\title{
Giro copernicano: Autoestima, capital psíquico e "inteligencia"
}

Fecha de aceptación: julio 2019

Martín Simonetta *

Resumen: El presente trabajo reflexiona en torno al vínculo existente entre el nivel de autoestima, el "capital psíquico" y la performance en la búsqueda de la felicidad de las personas. El concepto de "capital psíquico" ha sido desarrollado por Martina Casullo, referente de la escuela de psicología positiva en la Argentina, fundada a nivel global por Martin Seligman. Desde esta visión, la autoestima jugará un rol determinante a la hora de potenciar o restringir el logro de las metas de los individuos.

Es un gran honor la posibilidad de compartir estas reflexiones en el marco de la presente publicación de una institución tan prestigiosa e influyente como lo es la Universidad de Palermo. En este marco, nos referiremos a la forma en que la autoestima se vincula al capital psíquico de las personas y la forma en que potencian el uso del "stock neurológico", lo que habitualmente llamamos "inteligencia".

Palabras clave: Psicología positiva - intuición - autoestima - inteligencias múltiples - capital psíquico.

[Resúmenes en inglés y portugués en las páginas 114-115]

(*) Lic. en Relaciones Internacionales y Magister en Economía Política por la USAL. The Outstanding Young Person of Argentina-TOYP 2004 por Junior Chamber International y la Cámara Argentina de Comercio (CAC), Fue galardonado en 1999 con el British Chevening Scholarship por parte del British Council, la Embajada Británica y la Fundación Antorchas para desarrollar investigaciones en el marco del Institute of Economic Affairs (IEA), Londres, Gran Bretaña. En el 2004, la Fundación Friedrich Naumann le otorgó una beca para desarrollar un Programa de Liderazgo Internacional en Globalización y Regionalismo en la Theodore Heuss Akademie, sita Gümmersbach, Alemania. Es Director de Fundación Atlas y ha editado varios libros entre los cuales se encuentran "Políticas Liberales Exitosas" (2005, RELIAL, F. Naumann y F. Atlas 1853) y "Héroes de la Libertad" (2006, F. Hayek y F. Atlas 1853). Psicoeconomia. Economía de los sentimientos humanos. 


\section{Introducción}

Las presentes reflexiones se encuentran vinculadas que ver con mi obra "Psicoeconomía. Economía de los sentimientos humanos", publicada en 2014, que encuentra y trabaja en intersecciones entre ramas del saber, aparentemente, sin puntos de conexión: la psicología y la economía, así como también de la neurología (a partir del profundo aporte posibilitado por la actual tecnología al conocimiento del funcionamiento de nuestras redes neuronales).

\section{El reconocimiento reciente de la relevancia de los aspectos emocionales en la performance de las personas}

¿Quién es más “inteligente”? ¿El niño que más estudia? ¿El que logra mejores notas en la escuela? ¿El que cuenta con un mayor coeficiente intelectual? ¿Qué rol juegan los aspectos emocionales en el éxito de las personas?

Con la publicación de su "best-seller" Inteligencia Emocional, en 1995, el psicólogo Daniel Goleman generó un enorme impacto en amplios sectores de la sociedad. Goleman dedicó su obra a explicar por qué algunas personas parecen dotadas de un don especial que les permite vivir bien, aunque no sean las que más se destacan por su aparente nivel de inteligencia. Asimismo, por qué, por ejemplo, no siempre el alumno supuestamente "más inteligente" es el que termina siendo el más exitoso en alcanzar sus metas a lo largo de su vida. A través de tal obra, Goleman dotó de una enorme fuerza al concepto de inteligencia emocional, acuñado en 1990 por los psicólogos de la Universidad de Yale, Peter Salovey y John Mayer.

En las épocas previas, el enfoque predominante respecto de los "niveles de inteligencia" se centraba en la medición del coeficiente intelectual (CI o IQ, Coeficiente Intelectual / Intelligence Quotient), método desarrollado por Alfred Binet y Théodore Simon y utilizado inicialmente en 1912 por el psicólogo alemán William Stern a efectos de medir la capacidad intelectual en niños.

La teoría de la inteligencia emocional representó un giro copernicano que puso en jaque al entonces indiscutido criterio del CI/IQ. Existía una pregunta sin responder, un interrogante sin descifrar respecto de la relación entre la "inteligencia” y el rendimiento en la vida. ¿Por qué, en muchos casos, los supuestamente "menos inteligentes” triunfaban? O ¿por qué los "más inteligentes" no necesariamente alcanzaban sus metas?

El camino de la realidad parecía indicar que existía otra mirada posible al respecto, lo cual puede observarse en la reflexión de Goleman:

... uno de los secretos a voces de la psicología es la relativa incapacidad de las notas, el CI (coeficiente intelectual) o las pruebas de aptitud académica (SAT) para predecir de manera infalible quien tendrá éxito en la vida... El CI contribuye aproximadamente en un $20 \%$ a los factores que determinan el éxito en la vida, con lo que el $80 \%$ quedará para otras fuerzas. 
Estas “otras fuerzas", señaladas por Goleman, tenían que ver con factores de tipo emocional, poco considerados hasta ese momento. En su visión, la inteligencia emocional posibilita el desarrollo de habilidades tales como la capacidad de motivarse y persistir frente a decepciones, de controlar los impulsos, de generar esperanzas, de demorar la gratificación, de regular el humor, evitando que los trastornos disminuyan la capacidad de pensar. La inteligencia emocional permite al hombre ser consciente de sus emociones, entender los sentimientos de los demás, soportar las presiones y frustraciones, desarrollar capacidad de trabajar en equipo, así como adoptar una actitud empática y social que le brindará mayores posibilidades en el desarrollo de su persona.

Señala Goleman que el ser humano cuenta con dos tipos diferentes de inteligencias: la racional y la emocional, prevalecientes en diferentes hemisferios cerebrales. Mientras que el hemisferio izquierdo se enfoca en el procesamiento lógico de la información (a través del análisis, la realización de abstracciones, mediciones, planeamiento de procedimientos, verbalización, identificación de palabras y números, sumas, restas, lectura y escritura, etc.), el hemisferio derecho se especializa en la percepción global, dando un proceso de síntesis a la información que ingresa. Este hemisferio permite comprender y realizar metáforas, crear y/o combinar ideas. Cuenta con una visión holística, con capacidad de comprender lo global a partir de las partes. Es intuitivo, en lugar de lógico. Razona en imágenes, en símbolos, música y sentimientos. Este hemisferio también articula las relaciones interpersonales.

Esta comprensión del funcionamiento e interacción de ambos hemisferios cerebrales abrió nuevas puertas al entendimiento de lo que habitualmente llamamos "inteligencia". En términos de la teoría de las inteligencias múltiples desarrollada por Howard Gardner, el concepto de inteligencia emocional se vincula a las denominadas inteligencias interpersonal e intra-personal. La primera es la capacidad de establecer relaciones positivas con el entorno humano-social y la segunda se refiere a la capacidad de comprender los complejos procesos interiores que vivimos a nivel emocional.

\begin{tabular}{|l|l|}
\hline \multicolumn{2}{|c|}{ Las dos caras del cerebro } \\
\hline \multicolumn{1}{|c|}{ Hemisferio izquierdo (“racional”) } & \multicolumn{1}{c|}{ Hemisferio derecho (“emocional”) } \\
\hline $\begin{array}{l}\text { Verbal: Usa palabras para nombrar, describir, } \\
\text { definir. }\end{array}$ & $\begin{array}{l}\text { No verbal: Es consciente de las cosas, pero le } \\
\text { cuesta relacionarlas con palabras. }\end{array}$ \\
\hline $\begin{array}{l}\text { Analítico: Estudia las cosas paso a paso y parte } \\
\text { a parte. }\end{array}$ & $\begin{array}{l}\text { Sintético: Agrupa las cosas para formar } \\
\text { conjuntos. }\end{array}$ \\
\hline $\begin{array}{l}\text { Simbólico: Emplea un símbolo en representación } \\
\text { de algo. Por ejemplo, el dibujo un ojo; el signo } \\
+ \text { representa el proceso de adición. }\end{array}$ & $\begin{array}{l}\text { Concreto: Capta las cosas tal como son, en el } \\
\text { momento presente. }\end{array}$ \\
\hline $\begin{array}{l}\text { Abstracto: Toma un pequeño fragmento de } \\
\text { información y lo emplea para representar el todo. }\end{array}$ & $\begin{array}{l}\text { Analógico: Ve las semejanzas entre las cosas; } \\
\text { comprende las relaciones metafóricas. }\end{array}$ \\
\hline
\end{tabular}




\begin{tabular}{|c|c|}
\hline $\begin{array}{l}\text { Temporal: Sigue el paso del tiempo, ordena las } \\
\text { cosas en secuencias: empieza por el principio, } \\
\text { relaciona el pasado con el futuro, etc. }\end{array}$ & $\begin{array}{l}\text { Atemporal: Sin sentido del tiempo, centrado en } \\
\text { el momento presente. }\end{array}$ \\
\hline $\begin{array}{l}\text { Racional: Saca conclusiones basadas en la } \\
\text { razón y los datos. }\end{array}$ & $\begin{array}{l}\text { No racional: No necesita una base de razón, ni } \\
\text { se basa en los hechos, tiende a posponer los } \\
\text { juicios. }\end{array}$ \\
\hline Digital: Usa números, como al contar. & $\begin{array}{l}\text { Espacial: Ve donde están las cosas en relación } \\
\text { con otras cosas, y como se combinan las } \\
\text { partes para formar un todo. }\end{array}$ \\
\hline $\begin{array}{l}\text { Lógico: Sus conclusiones se basan en la lógica: } \\
\text { una cosa sigue a otra en un orden lógico. } \\
\text { Por ejemplo, un teorema matemático o un } \\
\text { argumento razonado. }\end{array}$ & $\begin{array}{l}\text { Intuitivo: Tiene inspiraciones repentinas, a } \\
\text { veces basadas en patrones incompletos, pistas, } \\
\text { corazonadas o imágenes visuales. }\end{array}$ \\
\hline $\begin{array}{l}\text { Lineal: Piensa en términos de ideas } \\
\text { encadenadas, un pensamiento sigue a } \\
\text { otro, llegando a menudo a una conclusión } \\
\text { convergente. }\end{array}$ & $\begin{array}{l}\text { Holístico: Ve las cosas completas, de una vez; } \\
\text { percibe los patrones y estructuras generales, } \\
\text { llegando a menudo a conclusiones divergentes. }\end{array}$ \\
\hline \multicolumn{2}{|l|}{ Fuente: Personarte.com } \\
\hline
\end{tabular}

\section{La autoestima determina qué porción del cerebro utilizamos}

El concepto de inteligencia emocional permitió comenzar a confirmar lo que por generaciones se intuyó: los aspectos emocionales posibilitan que el cerebro humano sea utilizado de forma óptima, al máximo, en su mayor potencial.

Si se me permite una metáfora del mundo productivo, así como una fábrica que podría producir 100 autos por día y sólo produce 10, un déficit en el terreno emocional podría hacer que las personas actúen sin utilizar al máximo sus potencialidades.

Resulta interesante observar cómo desde otro perfil científico, el de la ciencia económica, existe un abordaje similar -al de la inteligencia emocional- respecto de los factores que determinan el grado de utilización de los recursos. La teoría de la frontera de posibilidades de producción (conocida como FPP) explica la combinación de factores productivos o tecnologías en los que una unidad productiva alcanza su producción máxima. Es decir que refleja las cantidades máximas de bienes y servicios que un individuo, una empresa o una sociedad es capaz de producir en un determinado período de tiempo a partir de determinados factores de producción y conocimientos tecnológicos. Dado el uso de los factores productivos, encontramos tres escenarios posibles:

- Una estructura productiva ineficiente: cuando la producción real se encuentra por debajo de la producción máxima posible. Esto se puede dar porque no se utilizan todos 
los recursos, teniendo algunos de ellos capacidades ociosas. Como comentábamos en el ejemplo anterior, si en mi planta automotriz que me permite producir 100 autos diarios sólo produzco 10.

- Una estructura productiva eficiente: cuando el volumen de producción alcanza la producción máxima posible. En este caso, la existencia de recursos ociosos es mínima y el uso de tecnología no es mejorable. Produce 100 autos diarios de 100 posibles.

- Una estructura productiva inalcanzable: cuando lo producido se encuentra por encima de las posibilidades de producción. Este caso es teórico, ya que -por definición- es imposible. Los cambios tecnológicos hacen que esta frontera de producción se expanda y que lo imposible se transforme en posible. De esta forma, la imprenta que podía producir 5 libros por año en el 2000, en el año 2011 -con la incorporación de tecnología de diseño e impresión y manteniendo los demás factores constantes- podrá producir 500 libros. Ejemplos, similares podríamos realizar con la robotización aplicada a una fábrica de autos o la agricultura y el incremento de su productividad por hectárea como consecuencia de las semillas transgénicas.

\section{Frontera de posibilidades de producción (FPP)}

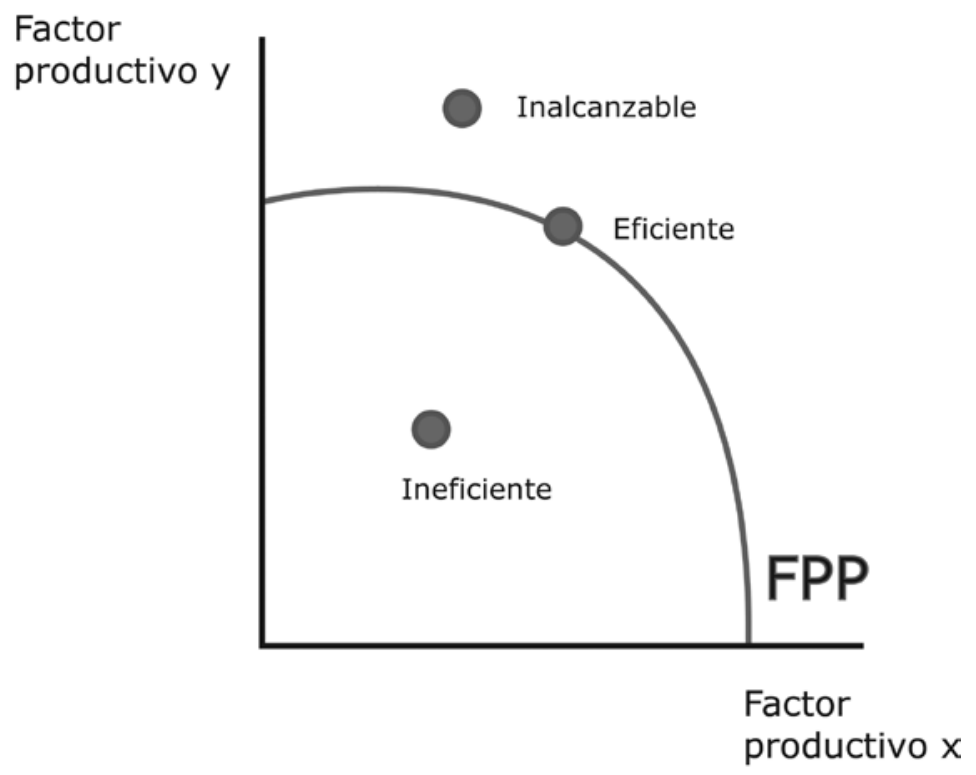


En términos generales, la Frontera de Posibilidades de Producción nos indica cuán lejos estamos de nuestra producción máxima posible.

Entonces, ¿qué tienen en común el concepto de inteligencia emocional y el de Frontera de Posibilidades de Producción? El concepto de Inteligencia Emocional nos dice que los aspectos emocionales juegan un rol fundamental en el desempeño de las personas. En consecuencia, es posible que un individuo con un coeficiente intelectual bajo, lo utilice al $100 \%$ y tenga un desempeño mayor al de otro con un coeficiente intelectual alto pero que lo utilice en un porcentaje menor.

Observemos un ejemplo en el siguiente cuadro: la persona A cuenta con un coeficiente intelectual inferior (2) al de la persona B (10). Sin embargo, la persona A utiliza esta menor dotación de recursos naturales en su máximo potencial (100\%), mientras que la persona B (la "más inteligente") la utiliza al 10\%. El resultado sorprende: la persona aparentemente menos inteligente utiliza más unidades (2) de inteligencia que la más inteligente (1).

¿Por qué sucede este milagro? Los aspectos emocionales son la clave. Ergo, las limitaciones del coeficiente intelectual no constituyen un límite, ni tampoco la abundancia es una garantía.

\begin{tabular}{|l|l|l|l|l|l|}
\hline \multicolumn{7}{|c|}{ ¿Quién es más inteligente? } \\
\hline & $\begin{array}{l}\text { Inteligencia } \\
\text { objetiva } \\
\text { (IQ o Cl, } \\
\text { Coeficiente } \\
\text { intelectual) }\end{array}$ & $\begin{array}{l}\text { Resultado } \\
\text { parcial }\end{array}$ & $\begin{array}{l}\text { Porcentaje } \\
\text { de uso de } \\
\text { inteligencia } \\
\text { objetiva como } \\
\text { consecuencia } \\
\text { de factores } \\
\text { emocionales }\end{array}$ & $\begin{array}{l}\text { Unidades } \\
\text { utilizadas de } \\
\text { inteligencia }\end{array}$ & $\begin{array}{l}\text { Resultado } \\
\text { final }\end{array}$ \\
\hline Persona A & 2 & $\begin{array}{l}\text { Menos } \\
\text { inteligente }\end{array}$ & $100 \%$ & 2 & Mayor \\
\hline Persona B & 10 & $\begin{array}{l}\text { Más } \\
\text { inteligente }\end{array}$ & $10 \%$ & 1 & Menor \\
\hline
\end{tabular}

En consecuencia, los aspectos emocionales jugarán un rol clave en determinar si la fábrica funcionará parcialmente o a plena capacidad. Cuanto mayor sea este "capital psíquico", mayor será el uso de la "capacidad productiva" de cada persona y menor la "capacidad ociosa". En consecuencia, las personas utilizarán un mayor porcentaje de su potencial, de sus "talentos". 


\section{Capital psíquico, análisis FODA y Psicología Positiva}

La historia de la psicología como ciencia ha recorrido un extenso camino. Sus orígenes, vinculados a la medicina, han estado enfocados en las psicopatologías, tales como la ansiedad, el stress, la depresión, por mencionar apenas algunas. En las últimas décadas, Martin Seligman -director del Departamento de Psicología de la Universidad de Pennsylvania habiendo sido previamente presidente de la American Psychological Association desde 1996- se erigió en el representante de una nueva tendencia teórica que no se enfocaría en las "enfermedades" psíquicas sino en las bases del bienestar psicológico y de la felicidad, así como en las virtudes y fortalezas humanas. De esta forma, dio origen a lo que hoy se conoce como la "Psicología Positiva".

El foco en los aspectos positivos de la psiquis humana significó un giro copernicano que ha puesto el énfasis en la identificación de las fortalezas y las virtudes de nuestro carácter, concentrándonos en ellas, reconociéndolas y desarrollándolas, como una forma de alcanzar una vida de plenitud. Estas fortalezas y virtudes son consideradas nuestro "capital psíquico", según el concepto desarrollado por María Martina Casullo, reconocida como la "madre" de la Psicología Positiva en la Argentina:

... los psicólogos identificados con el enfoque de la Psicología Positiva se interesan en comprender y analizar cómo y por qué, aún frente a situaciones de máximos estrés, los sujetos pueden desarrollar fortalezas, emociones positivas, proyectos de vida.

¿Qué factores, circunstancias y procesos intervienen para que la persona, a pesar de haber sufrido pérdidas, enfrentado conflictos, padecido enfermedades graves, considere que la vida merece ser vivida?

Se utiliza la noción de Capital Psíquico para hacer referencia a ese conjunto de factores y procesos que permiten aprender a protegerse y sobrevivir, a generar fortalezas personales.

Es interesante observar que el mundo de los negocios también ha desarrollado una herramienta similar -de gran simpleza y utilidad- para identificar "dónde estamos parados" y, de acuerdo a ello, desarrollar una estrategia de éxito. Nos referimos al análisis FODA -en inglés SWOT- cuya creación es atribuida a Albert Humphrey. Este método de análisis estratégico de las organizaciones nos permite identificar las Fortalezas y Debilidades dentro de nuestras empresas, así como las Oportunidades y Amenazas en el ambiente externo. Mientras las dos primeras variables (Fortalezas y Debilidades) son endógenas o internas a la organización, es decir que dependen de nosotros y las podemos modificar, las otras dos son exógenas o externas (Oportunidades y Amenazas) y no está en nosotros la capacidad de alterarlas.

Este análisis simple nos permite, en primer lugar, identificar sobre qué áreas podemos actuar y sobre qué áreas no lo podemos hacer. En consecuencia, nos aporta un enfoque absolutamente realista y nos permite concentrarnos en lo posible. La identificación y reconocimiento de "dónde estamos parados" constituye un primer y útil paso para poder actuar a partir de una estrategia realista en el logro de metas. Una de las recomendacio- 
nes empresarias emergentes es "enfocarse en fortalezas", lo cual implica -por ejemplo- la identificación de las unidades de negocios rentables y las no rentables, y desprenderse de estas últimas.

En su obra What You Can Change and What You Can't (Lo que usted puede cambiar y lo que no), Seligman hace referencia a la importancia estratégica de poder realizar esta diferencia. $\mathrm{Al}$ respecto, Catherine Meyer comenta la conocida pero no menos sabia reflexión: "Valor para cambiar lo que se puede cambiar. Serenidad para aceptar lo que no se puede cambiar. Sabiduría para reconocer la diferencia”.

Tan sencillo (y tan complejo) como eso.

\begin{tabular}{|c|c|}
\hline \multicolumn{2}{|r|}{$\begin{array}{l}\text { Clasificación de Virtudes y Fortalezas del Carácter } \\
\text { (Peterson \& Seligman, 2004) }\end{array}$} \\
\hline Virtudes & Fortalezas \\
\hline $\begin{array}{l}\text { Sabiduría y } \\
\text { sapiencia }\end{array}$ & $\begin{array}{l}\text { Creatividad (originalidad, inventiva); curiosidad (interés por el mundo, } \\
\text { búsqueda de novedad, apertura a la experiencia); mentalidad abierta } \\
\text { (capacidad de juicio, pensamiento crítico); amor por el conocimiento y } \\
\text { aprendizaje; perspectiva (sabiduría). }\end{array}$ \\
\hline Coraje & $\begin{array}{l}\text { Valentía (valor); persistencia (perseverancia, diligencia); integridad } \\
\text { (autenticidad, honestidad); vitalidad (pasión por las cosas, entusiasmo, vigor, } \\
\text { energía). }\end{array}$ \\
\hline Humanidad & $\begin{array}{l}\text { Amor; bondad o benevolencia (generosidad, calidez, cuidado, compasión, } \\
\text { amor altruista, amabilidad); inteligencia social (inteligencia emocional, } \\
\text { inteligencia personal). }\end{array}$ \\
\hline Justicia & Civismo (responsabilidad social, lealtad, trabajo en equipo); equidad; liderazgo. \\
\hline Templanza & $\begin{array}{l}\text { Capacidad de perdonar y misericordia; humildad y modestia; prudencia; auto- } \\
\text { regulación (autocontrol). }\end{array}$ \\
\hline Trascendencia & $\begin{array}{l}\text { Apreciación de la belleza y la excelencia (capacidad de asombro, admiración, } \\
\text { elevación); gratitud; esperanza (optimismo, proyección hacia el futuro, } \\
\text { orientación hacia el futuro); sentido del humor (humor positivo); espiritualidad } \\
\text { (religiosidad, fe, propósito). }\end{array}$ \\
\hline \multicolumn{2}{|c|}{$\begin{array}{l}\text { Fuente: Luciana Mariñelarena-Dondera \& Hugo Klappenbach, "La Psicología Positiva en la revista } \\
\text { Psicodebate (2000-2007)". Revista Psicodebate Nro. 10: Psicología, Cultura y Sociedad. Buenos } \\
\text { Aires, 2009. }\end{array}$} \\
\hline
\end{tabular}

La visión de Goleman aporta claridad a una sensación intuida a lo largo de la historia de la humanidad: el valor estratégico de los aspectos emocionales. La historia afectiva de la persona se inicia desde su misma concepción e incluso cuenta con fuertes determinantes ge- 
néticos. En este contexto, el rol de lo emocional jugará un papel central en el afianzamiento de la personalidad, la consolidación de su autoestima, así como en el éxito en la vida.

Estudios como los de John Bowlby señalan que el proceso de constitución de este capital emocional tiene una vinculación fundamental con la experiencia de los primeros años de vida de las personas. Bowlby en 1958 publicó The Nature of the Child's Tie to his Mothe (La naturaleza del vínculo del niño con su madre) que fue la base de su teoría del "apego" ("attachment") referida a la forma en que el vínculo entre los niños y sus padres (naturales o por adopción) tiene un rol clave en el desarrollo del capital emocional que se podrá observar "en acción" a lo largo de su vida posterior. Esta teoría del apego se confirma también en otros animales además de los humanos, tanto en aves como en otros mamíferos. La proximidad bebé-madre, el contacto físico, y la protección constituyen cimientos fundamentales en la construcción del edificio afectivo.

La particular necesidad de cuidado al nacer por parte del bebé humano (por encima de otras especies) tendría que ver con una de sus grandes virtudes diferenciales: el tamaño de su cerebro. Elizabeth Antunovic se ha referido al concepto de "extero-gestación" o gestación externa que hace que -los homos sapiens- nazcamos "antes de lo previsto" debido al gran tamaño de nuestro cerebro. $\mathrm{Al}$ respecto afirma:

- Para que un bebé humano alcanzara la mitad del tamaño de un cerebro adulto tardaría unos 18 meses de gestación.

- El bebé humano nace usualmente 266,5 días después de la concepción debido a su cabeza grande y al rápido crecimiento de su cerebro que tiene lugar durante los últimos tres meses en el útero.

- Los humanos nacen con un 25\% del cerebro de un adulto, los simios el 50\%.

- Los bebés humanos nacen también inmaduros. De hecho, siguen siendo en realidad más desvalidos que las crías de cualquier otra especie.

Tanto la visión de Goleman (Inteligencia emocional), como la de Seligman (Fortalezas y virtudes del carácter) y Martín Casullo (Capital psíquico) parecen coincidir en el determinante rol de lo emocional a la hora de influir en el rendimiento de las personas en el proceso de búsqueda de su felicidad. En "producir" su felicidad en su máximo potencial o no, alcanzando el límite máximo de sus "fronteras de posibilidades de producción" e incluso ampliándolas.

\section{Inteligencias múltiples}

El escape de la visión monocorde de la inteligencia tuvo un punto de inflexión en 1983, cuando el neuropsicólogo Howard Gardner publicó su libro Frames of Mind: The Theory of Multiple Intelligences, (Marcos de la Mente: La Teoría de las Inteligencias Múltiples) que lo hizo precursor en el cuestionamiento del criterio de coeficiente intelectual cómo único parámetro, afirmando que no existía una única y monolítica clase de inteligencia.

¿Podemos comparar las inteligencias de Leonardo da Vinci, Luciano Pavarotti, Diego Maradona, Lionel Messi, Steve Jobs, Niccolò Machiavelli, Bill Gates, Albert Einstein, Wolfgang 
Amadeus Mozart, Jesucristo, Freddie Mercury, Walt Disney, Nelson Mandela, Sigmund Freud o Adam Smith? ¿Podemos afirmar que alguno de ellos es/era más inteligente que los demás?

De acuerdo a Gardner cada una de estas personas poseería un tipo específico de inteligencia. En su visión, la inteligencia es la capacidad de ordenar los pensamientos y coordinarlos con las acciones, pero no es una unicidad. Cada individuo cuenta con al menos siete inteligencias, habilidades cognitivas, que trabajan conjuntamente, pero de forma semi-autónomas. Cada persona desarrolla algunas más que otras, y diferentes culturas y segmentos de la sociedad ponen diferentes énfasis en cada una de ellas.

Las siete inteligencias señaladas por Howard Gardner (luego se añadió una octava inteligencia: la ambiental) son las siguientes:

1. Lingüística. La habilidad para leer, escribir y hablar bien. Característica de escritores y oradores, por ejemplo.

2. Lógica-matemática. La habilidad de resolución de problemas.

3. Corporal y kinestésica. Característica de los deportistas.

4. Visual y espacial. La habilidad para moverse y navegar en el espacio y la habilidad para desarrollar escenas tridimensionales en la mente.

5. Musical. Habilidad natural para leer, escribir y tocar instrumentos musicales.

6. Intra-personal. La habilidad de comprender las propias, motivaciones, emociones y otros aspectos de la propia personalidad.

7. Inter-personal. La capacidad de comprender a los demás. El concepto de "empatía" como comprensión de las emociones de las personas. El don que nos permite ponernos en lugar del otro, comprendiendo sus motivaciones y emociones.

Leonardo da Vinci, ícono del hombre renacentista, es un ejemplo de persona con una performance destacada en diversos ámbitos que parecen hasta opuestos, como lo son el arte y las ciencias. Pintó la Gioconda, realizó grandes avances en la medicina y hasta se animó a imaginar el avión muchos siglos antes de que su implementación fuera técnicamente posible. A este tipo de personalidades destacadas en distintas ramas del saber se las denomina también "polímatas".

Como hemos comentado anteriormente, los conceptos de inteligencia "inter-personal" e "intra-personal" de Gardner son muy cercanos a los de "inteligencia emocional” de Goleman. Al respecto afirma en Multiple Intelligences:

La inteligencia inter-personal es la capacidad para comprender a los demás: qué los motiva, cómo operan, cómo trabajar cooperativamente con ellos. Vendedores, políticos, maestros, médicos clínicos y líderes religiosos de éxito tienen probabilidades de ser individuos con elevado grado de inteligencia interpersonal. La inteligencia intra-personal... es una capacidad correlativa, vuelta hacia el interior. Es la capacidad de formar un modelo preciso y realista de uno mismo y ser capaz de usar ese modelo para operar eficazmente en la vida. 


\section{La ventaja emocional de las personas}

Si hablamos de capital psíquico y que este varía de persona a persona, podremos decir que habrá individuos con mayor capital psíquico que otros.

A principios del siglo XIX, uno de los padres de la economía, David Ricardo, desarrolló la teoría de la "ventaja comparativa" que afirmaba que las naciones debían enfocarse en producir aquello que hicieran mejor, más eficientemente. Más recientemente, desde una perspectiva de negocios, Michael Porter elaboró el concepto de "ventaja competitiva" de las naciones. En su visión, la comprensión de su potencial estratégico (tierra, ubicación, recursos naturales, mano de obra, tamaño de la población) y un accionar basado en ello, constituyen la clave para el éxito económico de los países.

Siguiendo este razonamiento, nos preguntamos si podemos pensar en una "ventaja emocional" de las personas, una "superioridad relativa" consecuencia de su mayor capital psíquico. ¿Tienen las personas con un mayor desarrollo de su capital psíquico mayores posibilidades de alcanzar sus metas a lo largo de la vida? ¿Pueden, incluso, incrementar este capital a través de la comprensión de los procesos que lo determinan?

Para finalizar estas reflexiones, nos preguntamos: ¿Cuál es el máximo potencial de cada persona? ¿Cuál el mayor rendimiento posible de cada uno de nosotros para alcanzar la meta que nos fijemos? Citando una expresión muy utilizada en los Estados Unidos, podríamos decir que "el cielo es el límite" (the sky is the limit). El desarrollo de nuestro capital psíquico es un determinante en el corrimiento del tope de nuestra frontera de posibilidades de producción, de nuestro rendimiento en los diferentes aspectos que nos interesen a lo largo de las diferentes etapas de nuestra de vida.

Aún en situaciones de personas que parezcan contar con un "menor nivel de inteligencia", contextos adecuados permitirán la generación y desarrollo de un capital psíquico. Políticas como la de la cadena de comidas rápidas Mc Donald's de contar con un cupo de trabajo para jóvenes con síndrome de Down son muestras de que estas personas pueden alcanzar un rendimiento laboral equivalente al del resto de las personas en muchas áreas. En un contexto de inteligencias múltiples, las capacidades diferenciadas sustituyen al concepto de discapacidad, e incluso las personas consideras "normales" contaríamos con capacidades o discapacidades múltiples.

En situaciones de extrema adversidad, será este capital psíquico el que permita a algunas personas recuperarse, re-inventarse y volver a emprender su camino con más fuerza. Este proceso denominado resiliencia es característico de grandes personalidades de la historia de la humanidad tales como Alexander Graham Bell, quien contribuyó fuertemente en la invención del teléfono, obsesionado por los problemas auditivos de su madre y su esposa. La historia de la humanidad es -afortunadamente- rica en este tipo de ejemplos. Si miramos a nuestro derredor cercano, es posible que encontremos decenas de casos.

El proceso de resiliencia o recuperación se puede observar incluso en el terreno neurológico, aún en situaciones de graves traumatismos o patologías cerebrales. Si bien tiempo atrás prevalecía la idea de que las neuronas no se reproducen y constituyen un "stock" fijo que no puede incrementarse, sino tan sólo reducirse en su número, los avances de la ciencia modificaron tal supuesto. Al respecto resultan interesantes los estudios de Elkhonon Goldberg, neurólogo de New York University y director del Instituto de Neuropsicología y 
Funcionamiento Cognitivo, que -conjuntamente con estudios de otros científicos- ponen en evidencia que la regeneración cerebral (nuevas neuronas y nuevas conexiones neuronales en las áreas más estimuladas) es posible y que este órgano cambia de forma según su utilización. Esto es lo que se denomina "neuroplasticidad".

Nuestro cerebro es "flexible" y, así como en la actividad física, los músculos que más usamos son los que más se desarrollan, lo mismo parece suceder con este órgano. De ahí el nacimiento del concepto de entrenamiento cognitivo o "fitness mental". Como señala Néstor Braidot, el cerebro es como un músculo y debemos mantenerlo activo y su ejercitación no sólo mejora las funciones cognitivas, sino que genera modificaciones en este órgano clave. Contrariamente, la ausencia de actividad atenta contra su buen funcionamiento.

La teoría de la inteligencia emocional esbozada por Goleman revolucionó la concepción de prevaleciente hasta el momento en la materia, transformó lo estático en dinámico, y reconoció a los aspectos emocionales su verdadera y profunda relevancia.

\section{Bibliografía}

Braidot, N. (2008). Neuromanagement. Cómo utilizar a pleno el cerebro en la conducción exitosa de las organizaciones, p. 47. Biblioteca Braidot, Ediciones Granica.

Gardner, H. "Multiple Intelligences", citado por Daniel Goleman en La inteligencia emocional. Por qué es más importante que el cociente intelectual, p. 60, op. cit.

Porter, M. (1985). Ventaja competitiva. Creación y sostenimiento de un desempeño superior.

"Psicoeconomía. Economía de los sentimientos humanos", escrita por Martín Simonetta y publicada por la Fundación Foro para la Salud Mental, 2014.

Casullo, M. M. (2005). "El capital psíquico. Aportes de la Psicología Positiva”. Revista Psicodebate No 6: Psicología y Salud. Buenos Aires.

Gardner, H. (1998). "A Reply to Perry D. Klein's 'Multiplying the problems of intelligence by eight". Canadian Journal of Education 23.

Agüera Torres, A. (2009). "División de actividades por inteligencia en educación infantil". Revista digital Enfoques Educativos No 32, 15 de febrero.

Bowlby, J. (1958). “The Nature of the Child `s Tie to his Mother». International Journal of Psychoanalysis, 39: 350-371.

Antunovic, E. (2009). El mejor lugar después del vientre materno. La exterogestación y la necesidad de ser llevado.

Goleman, D. (1999). La inteligencia emocional, p. 54. Vergara Editor.

\footnotetext{
Abstract: This paper reflects on the link between the level of self-esteem, "psychic capital" and performance in the pursuit of happiness of people. The concept of "psychic capital" has been developed by Martina Casullo, referring to the school of positive psychology in Argentina, founded on a global level by Martin Seligman. From this perspective, self-esteem will play a determining role when it comes to strengthening or restricting the achievement of individuals' goals.
} 
It is a great honor to share these reflections within the framework of the present publication of an institution as prestigious and influential as the University of Palermo. In this framework, we will refer to the way in which self-esteem is linked to the psychic capital of people and the way in which they enhance the use of the "neurological stock", which we usually call "intelligence".

Keywords: Positive psychology - intuition - self-esteem - multiple intelligences - pisquico capital.

Resumo: Este artigo reflete sobre a relação entre o nível de autoestima, "capital psíquico" e desempenho na busca da felicidade das pessoas. O conceito de "capital psíquico" foi desenvolvido por Martina Casullo, referindo-se à escola de psicologia positiva na Argentina, fundada em nível global por Martin Seligman. Nessa perspectiva, a autoestima terá um papel determinante no fortalecimento ou restrição do alcance dos objetivos dos indivíduos. É uma grande honra compartilhar essas reflexões no âmbito da atual publicação de uma instituição tão prestigiosa e influente quanto a Universidade de Palermo. Nesse contexto, vamos nos referir ao modo como a auto-estima está ligada ao capital psíquico das pessoas e à maneira como elas intensificam o uso do "estoque neurológico", que geralmente chamamos de "inteligência".

Palavras chave: Psicologia positiva - intuição - auto-estima - inteligências múltiplas - capital pisquico.

[Las traducciones de los abstracts fueron supervisadas por el autor de cada artículo] 